PharmacoEconomics \& Outcomes News 884, p34 - 7 Aug 2021

\title{
UK's NICE issues guideline on diagnosing rare COVID-19 vaccination- related blood clots
}

UK's National Institute for Health and Care Excellence (NICE) has published new rapid guideline to help identify and treat patients who develop a rare blood-clotting syndrome after receiving COVID-19 vaccinations.

Vaccine-induced immune thrombocytopenia and thrombosis (VITT) is extremely rare, with only 14.2 cases per million doses of COVID-19 vaccine, but can be very serious and requires swift diagnosis and treatment.

The new guideline helps healthcare staff to identify people with suspected VITT, advises which tests should be used to confirm the diagnosis, and covers treatment options for people with VITT according to treatment severity and response.

Commenting on the guidelines, Director of NICE's Centre for Guidelines, Dr Paul Chrisp, said: "although VITT is a very rare condition, it's crucial that healthcare professionals feel supported and able to swiftly identify and treat the small number of people who do develop it. This is a living guideline, which can be continuously updated to incorporate the latest evidence and keep abreast of new developments."

National Institute for Health and Care Excellence (NICE). NICE publishes new rapid guideline to diagnose and treat rare blood clotting condition associated with COVID-19 vaccination Internet Document : 3 Aug 2021. Available from: URL: https://www.nice.org.uk/news/article/nice-publishes-new-rapid-guideline-to-diagnose-and-treat-rare-bloodclotting-condition-associated-with-covid-19-vaccination 